Introduction Parkinson's disease (PD) is a neuro-degenerative disorder with frequent involvement of the gut. Symptoms arise throughout the gastrointestinal tract through dysmotility secondary to autonomic and enteric nervous system involvement, as well from skeletal muscle involvement in the oropharynx and anorectum. It has been speculated that gut involvement may precede motor symptoms. The Wireless Motility Capsule (WMC) yields data on transit and motility throughout the gut. We report the first use of WMC to systematically assess motility in PD patients with and without gut symptoms, compared to controls. Methods 15 patients with established PD completed the study: eight ( $2 \mathrm{f}$, mean age 70 [47-85]) had GI symptoms and seven ( $2 \mathrm{f}$, mean age 61 [49-77]) did not based on history and baseline scores on the Gastroparesis Cardinal Symptom Index (GCSI) and Wexner constipation score. Data comparison with seven controls (3f, mean age 52 [39-63]). Medications affecting GI motility / $\mathrm{pH}$ were discontinued for the study and the WMC was ingested following a standardised nutrient bar meal. Data on gastric emptying time (GET), small bowel transit time (SBTT), colonic transit time (CTT) and whole gut transit time (WGTT) were calculated.

Results PD patients with gut symptoms showed significantly slower transit in the stomach (GET 5.2 vs. $2.7 \mathrm{~h}, \mathrm{p}=0.0003$ ), colon (CTT 57.8 vs. $27.4 \mathrm{~h}, \mathrm{p}=0.02$ ) and overall gut (WGTT 67.2 vs. $34.7 \mathrm{~h}, \mathrm{p}=0.02)$ compared to asymptomatic patients. Small Bowel transit (mean SBTT $4.17 \mathrm{~h}$ ) did not significantly differ. GET, SBTT, CTT and WGTT did not differ between asymptomatic PD and controls. There was a significant correlation between the Wexner constipation score and CTT in all patients $(\mathrm{p}<0.01)$, but no correlation between GCSI and gastric emptying $(\mathrm{p}>0.05)$.

Conclusion This study demonstrates that symptomatic PD patients have markedly delayed transit times throughout the whole gut compared to asymptomatic PD patients and controls. The correlation between scores and transit times suggest that WMC is a less useful indicator of gastric emptying than small bowel and colonic transit.

Disclosure of Interest None Declared.

\section{PWE-183 PRELIMINARY SIGNIFICANT FINDINGS FROM A RANDOMISED CONTROL TRIAL OF POSTERIOR TIBIAL NERVE STIMULATION IN SYSTEMIC SCLEROSIS ASSOCIATED FAECAL INCONTINENCE}

${ }^{1}$ SK Butt*, ${ }^{*} \mathrm{~A}$ Alam, ${ }^{1} \mathrm{~A}$ Raeburn, ${ }^{1} \mathrm{~J}$ Liwanag, ${ }^{2} \mathrm{VH}$ Ong, ${ }^{2} \mathrm{CP}$ Denton, ${ }^{3} \mathrm{CD}$ Murray, ${ }^{1} \mathrm{~N}$ Zarate-Lopez, ${ }^{1} \mathrm{~A}$ Emmanuel. 'Gastroenterology, UCL London, London, UK; ${ }^{2}$ Rheumatology, Royal Free Hospital, London, UK; ${ }^{3}$ Gastroenterology, Royal Free Hospital, London, UK

\subsection{6/gutjnl-2014-307263.443}

Introduction The gastrointestinal tract is affected in up to $90 \%$ of Systemic Sclerosis (SSc) patients with faecal incontinence (FI) being reported in up to $38 \%$. Passive faecal incontinence secondary to internal anal sphincter atrophy is the characteristic finding. We have shown that neuropathic changes are implicated in SSc patients with FI and sacral nerve stimulation has emerged as a potentially beneficial therapy in SSc. However this is expensive, invasive, not widely available and we have shown that medium term efficacy is poor. Posterior tibial nerve stimulation (PTNS) is a potential alternative to modulate the sacral plexus indirectly, with none of these disadvantages. This is the preliminary data on a randomised placebo controlled trial of PTNS versus sham PTNS to determine if nerve modulation is an effective treatment in SSc associated FI.
Methods We commenced a prospective randomised single-blind study of SSc patients with FI in February 2013 from a specialist Scleroderma unit. Baseline symptom scoring (bowel diary, Wexner), manometry and endoanal ultrasound were completed prior to randomization to PTNS or sham. PTNS was administered conventionally, by insertion of an acupuncture needle according to anatomical landmarks, connected to an electrical stimulator. Sham PTNS was administered in identical fashion but the PTNS surface electrode was not connected and instead separate TENS surface electrodes were connected to a TENS unit. Each patient underwent blinded intervention for 30 min periods, once a week for 12 weeks. The primary endpoints were the percentage reduction in faecal incontinence episodes and change in Wexner incontinence scores.

Results A total of 13 SSc patients (11 f), mean age 61 (36-72) completed the trial by October 2013. Of these 6 (5 f) underwent PTNS and 7 (6 f) patients underwent sham stimulation. All PTNS patients showed a reduction $(5-100 \%)$ in the number of FI episodes in comparison to 0 sham patients at 12 weeks ( $\mathrm{p}<$ 0.01 (CI: -81.49-14.34)). This matched an improvement in mean Wexner scores from baseline to treatment end (14.8 to 10.8 vs 13.4 to 13.6 , true vs sham respectively, $\mathrm{p}=0.03$.

Conclusion This pilot data is demonstrating significant effects of PTNS in Scleroderma-associated FI. We present this significant initial data but anticipate having at least 25 completed patients by May 2014.

Disclosure of Interest None Declared.

\section{PWE-184 THE EFFECT OF FIBRE ON CHRONIC CONSTIPATION IN ADULTS: A SYSTEMATIC REVIEW}

${ }^{1} S$ Christodoulides*, ${ }^{2}$ E Dimidi, ${ }^{3} \mathrm{KC}$ Fragkos, ${ }^{4} \mathrm{PG}$ McLean, ${ }^{1} \mathrm{Q}$ Aziz, ${ }^{2} \mathrm{~K}$ Whelan, ${ }^{1} \mathrm{SM}$ Scott. ${ }^{1}$ Neurogastroenterology Group, Queen Mary University of London, London, UK; ${ }^{2}$ Diabetes and Nutritional Sciences Division, King's College London, London, UK; ${ }^{3}$ Centre for Gastroenterology and Clinical Nutrition, University College London, London, UK; ${ }^{4}$ Nestlé Research Centre, Lausanne, Switzerland

\subsection{6/gutjn--2014-307263.444}

Introduction Symptoms of chronic constipation are common, with a prevalence of $\sim 14 \%$ in adults worldwide. Although increasing fibre intake is universally accepted as a first-line treatment, patient dissatisfaction is common. A systematic review is thus required in order to evaluate the quality of scientific evidence behind this management approach. The aim was to assess the effect of fibre on chronic constipation and IBS-C in adults via a systematic review of randomised controlled trials (RCTs). Methods Following Cochrane and PRISMA recommendations, references were identified by searching 5 electronic databases, hand-searching abstracts of 3 annual conferences, as well as scanning reference lists. Two reviewers independently assessed all studies identified, and performed data extraction and risk of bias assessment. Only RCTs reporting administration of fibre (carbohydrate polymers with $\geq 3$ monomers that are not hydrolysed by endogenous enzymes in the small intestine) in adults with either chronic constipation or IBS-C were included.

Results Of 916 records identified, only 9 RCTs were eligible (495 patients). Fibre supplements, administered in the form of powder, granules, yoghurt or milk, were: psyllium $(\mathrm{n}=3)$, galacto-oligosaccharides (GOS: $\mathrm{n}=2$ ), wheat bran $(\mathrm{n}=1)$, inulin $(\mathrm{n}=1)$, and mixtures of inulin/ resistant maltodextrin $(\mathrm{n}=1)$, and inulin/partially hydrolysed guar gum $(n=1)$. Outcomes measured included stool frequency $(\mathrm{n}=9)$, stool consistency ( $\mathrm{n}$ $=6)$, stool weight $(\mathrm{n}=5)$, faecal microbiota $(\mathrm{n}=3)$ and 